Литература

1. Жуковский Э.И. Реализация задачи оценивания показателей работы завода в АСУТП сахарного производства/ Э.И. Жуковский, Ю.М. Скаковский, В.Д. Витвицкий, А.В. Бабков // Автоматизация технологических и бизнес-процессов. - Одесса: 2010 - №3.- С.16-26.;

2. Вітвіцький В.Д. Рішення задач оперативного обліку в АСУТП ділянок цукробурякового виробництва/ В.Д. Вітвіцький, Ю.М. Скаковський // Наук. пр. Одес. нац. акад. харч. техн./ Міністерство освіти і науки України. - Одеса: 2004 -Вип. 27.- С.213-221.

Referens

1. Gukovskiy E.Y. Realizacia zadachi ocenivania pokazateley raboti zavoda v ASUTP saharnogo proizvodstva/ E.Y. Gukovskiy, Y.M. Skakovskiy, V.D. Vitvitskiy, A.V. Babkov //Avtomatizacia tehnologicheskih i biznesprocesov.- Odessa:2010 - №3.- S.16-26.;

2. Vitvitskiy V.D. Rishennia zadach operativnogo obliku v ASUTP dilianok cukroburiakovogo virobnictva/ V.D. Vitvitskiy, Y.M. Skakovskiy //Nauk. pr. ONAHT/Ministerstvo osviti Ukraini.- Odesa: 2004.- Vip.27.- S.213221.

\title{
РАЗРАБОТКА МОДЕЛИ И ПРОГРАММНЫХ СРЕДСТВ ДЛЯ СОЗДАНИЯ РОБАСТНОЙ СИСТЕМЫ УПРАВЛЕНИЯ ТЕПЛООБМЕННИКОМ
}

Development of the model and software for heat exchanger robust control system design

Стопакевич A. O. ${ }^{1}$ (Stopakevych A. O.)

${ }^{1}$ Одесская национальная академия связи им. Попова, Одесса, E-mail: stopakevich@gmail.com

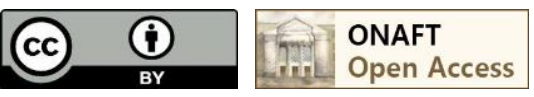

DOI: $10.15673 /$

\begin{abstract}
Введение
В статье рассматривается построение математической модели, а также структура математического и программного обеспечения для синтеза робастных систем автоматического управления парожидкостными теплообменниками. Показана актуальность задачи и описаны трудности её решения. Отличием предложенного алгоритма построения модели теплообменника является минимальное число требуемых исходных данных: начальная и конечная температуры жидкости, массовый расход жидкости и давление в паропроводе. Алгоритмизируется конструктивный расчет, расчет потерь, расчет регулирующего органа, расчет модели динамики теплообменника, расчет модели динамики датчика температуры, расчет регулятора с учетом робастности замкнутой системы автоматического управления. Алгоритм конструктивного расчета позволяет выбрать стандартный теплообменник. Программное обеспечение реализует разработанные алгоритмы. Анализ переходных процессов, полученных при ряде нагрузок теплообменника, доказывает, что разработанная система автоматического управления является робастной.
\end{abstract}

\section{Abstract}

The article describes the development of a mathematical model, mathware and software for steam-liquid heat exchanger robust control system design. The article spotlights the problem actuality and difficulties of its solving. The advantage of proposed algorithm for the exchanger model design is a minimal number of input variables, namely initial and final temperature of the liquid, mass flow rate of the liquid, pressure in the steam line. The algorithm includes constructive design, calculation of losses, calculation of the control valve, calculation of the heat exchanger dynamic model, calculation of the temperature sensor dynamic model and design of robust controller for the closed-loop control system. The algorithm of constructive design allows choosing a heat exchanger from 
standard line. The software implements developed mathware. The analysis of transient processes of closed-loop control system worked under the set of the exchanger loads proves the robustness of the system

Ключові слова

Теплообменник парожидкостный, математическая модель, статика, динамика, робастность, система автоматического управления.

\section{Постановка задачи}

Активное развитие подходов к решению задачи разработки аналитически моделей и программных средств для расчета параметров технологических установок в странах с передовой промышленностью идет в трех основных направлениях: разработка универсальных программных систем технологического моделирования, разработка специализированных языков для моделирования технологических процессов, разработка специализированного программного обеспечения на языках общего назначения для расчета конкретных технологических аппаратов.

Первое направление представлено такими программами технологического моделирования, как HYSYS/UniSim и CHEMCAD [1]. Несмотря на универсальность и ориентацию на сложные установки, расчет с их помощью в ряде случаев оказывается очень неточным, особенно это касается описания динамики объектов [2,3]. Исследование показало, что работают программы неудовлетворительно при создании системы автоматического управления (CAУ) даже такого относительно простого, широко распространенного аппарата, как парожидкостный кожухотрубный теплообменник (КТ).

Как специализированный язык для расчета и моделирования технологических процессов исследован язык Modelica [4]. Реальное его использование по нашему мнению целесообразно только при использовании коммерческих библиотек компонентов, точность и адекватность которых является предметом отдельного исследования.

Как специализированное программное обеспечение исследована программа Xchanger Suite фирмы HTRI [5]. Результаты расчета этой программы принимаются многими международными производителями теплообменников. Недостатком её для Украины является ориентация на конструктивные требования, записанные в международном стандарте организации Tubular Exchanger Manufacturer Association (TEMA), который не совпадает с ГОСТ. Это приводит к определенным отличиям в методиках расчета [6]. Кроме того, в программе проводится расчет исключительно статики, что недостаточно для полного расчета системы автоматического управления (САУ) КТ.

Итак, заключаем, что программное обеспечение, для проведения полного расчета САУ КТ совместно с расчетом модели теплообменника, отсутствует и задача его разработки является актуальной.

В качестве средства разработки для создаваемого ПО выбрана программная система Маtlab фирмы Mathworks, которая позволяет разрабатывать программное обеспечение с графическим интерфейсом, используя сложные математические алгоритмы. В состав Matlab входит подсистема физического моделирования Simscape, позволяющая реализовать аналогичный Modelica подход к моделированию физических систем. Более того, распространенность Matlab и большое количество его пользователей позволило разработать множество специализированных программных библиотек, таких как пакет термодинамических расчётов Тhermolib, библиотека для определения термодинамических постоянных по стандарту IAPWS IF-97 Xsteam [7], библиотека моделирования анализа и термодинамических систем от NASA T-MATS, интерфейсы к библиотекам термодинамических компонентов от NIST TDE и т.д.

\section{Структура и параметры ПО}

Схема укрупненного алгоритма полного расчета КТ приведена на рис. 1. Как видно, расчет статики и конструктивный расчет производится на основании заданных расхода жидкости $m_{2}$, требуемого перепада температур жидкости (воды) $t_{2 i}, t_{2 o}$ и давления в паропроводе $P_{s}$. Результатом конструктивного расчета является выбор теплообменника из стандартного ряда. На основании диаметра штуцера теплообменника и гидравлического расчета потерь рассчитываются параметры регулирующего органа на паропроводе в соответствии с РМ4-163-77. Затем рассчитывают параметры модели динамики, на основании которой можно получить адекватные настройки ПИД регулятора и провести моделирование его работы с математическими моделями средств автоматизации. 


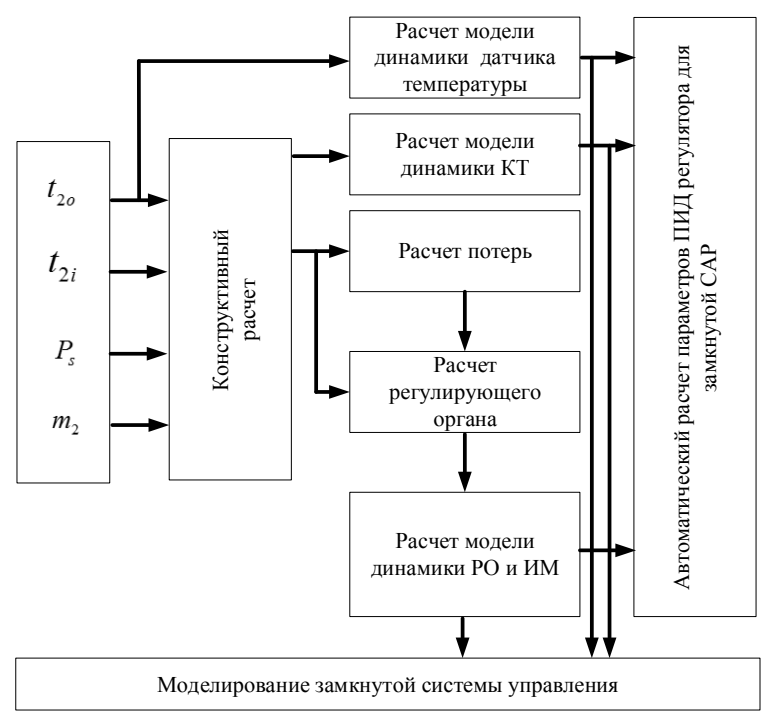

Рис. 1 - Схема укрупненного алгоритма полного расчета КТ

ПО состоит из двух компонентов: программного класса ShellAndTubeHeatExchanger и модели в системе Simulink с графическим интерфейсом, который реализован в среде GUIDE.

Входными параметрами конструктора программного класса являются: начальная температура $t_{2 i}\left({ }^{\circ} C\right)$, конечная температура $t_{2 o}\left({ }^{\circ} \mathrm{C}\right)$ и расход $m_{2}\left(\right.$ кг/с) нагреваемой жидкости, давление в паропроводе $P_{s}(\mathrm{MПа)}$.

Параметры, значения которых определяются путем последовательного приближения в конструктивном расчете, являются: скорость движения воды $w_{2}$ (м/с), внутренний диаметр труб КТ $d_{i}$ (м), внешний диаметр труб КТ $d_{o}$ (м), длина трубы КТ $L$ (м), к.п.д. КТ $\eta$, плотности тепловых потоков $q_{1}$ і $q_{2}($ Вт/м²), коэффициенты теплоотдачи от пара к стенке $\alpha_{1}$ и от стенки к жидкости $\alpha_{2}\left(\mathrm{BT} / \mathrm{M}^{2}\right)$.

Табличными данными, которые используются в расчетах являются коэффициент теплового расширения жидкости $\beta\left({ }^{\circ} \mathrm{C}^{-1}\right)$, температура насыщенного пара $t_{l}\left({ }^{\circ} \mathrm{C}\right)$, в зависимости от давления, удельная теплоемкость воды в зависимости от температуры - $C_{2 i}$ и $C_{2 o}$ (Дж/(кг $\left.{ }^{\circ} \mathrm{C}\right)$ ), удельная теплоемкость металла труб $C_{w}$ $\left(\right.$ Дж/(кг. $\left.\left.{ }^{\circ} \mathrm{C}\right)\right)$, удельная теплота парообразования $r_{1}($ Дж/кг) в зависимости от температуры, кинематическая вязкость жидкости $v_{2}\left(\mathrm{~m}^{2} / \mathrm{c}\right)$ в зависимости от температуры, теплопроводность жидкости $\lambda_{2} \quad(\mathrm{BT} / \mathrm{M} \cdot \mathrm{c})$, теплопроводность металла труб $\lambda_{w}(\mathrm{BT} / \mathrm{M} \cdot \mathrm{c})$ динамическая вязкость в зависимости от температуры $\mu$ (Па·с), плотности конденсата и насыщенного пара $\rho_{1}{ }^{\prime}, \rho_{1} "$ (кг/м $\left.{ }^{3}\right)$ в зависимости от температуры, плотность нагреваемой

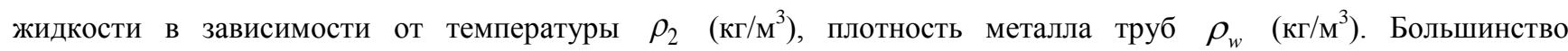
перечисленных данных могут быть получены с помощью библиотеки термодинамических параметров воды и пара Xsteam [7].

Основными результатами конструктивного расчета являются средний температурный напор LMTD $\left({ }^{\circ} \mathrm{C}\right)$, массовый расход пара $m_{l}(\kappa г / \mathrm{c})$, расчётный коэффициент теплопередачи $U p\left(\mathrm{BT} / \mathrm{M}^{2} \mathrm{~K}\right)$, расчётная поверхность теплообмена $A p\left(\mathrm{M}^{2}\right)$ и табличные параметры модели теплообменника с ближайшей большей поверхностью теплообмена (поверхность теплообмена $A$, диаметр кожуха $D o$, длина труб $L$, количество труб $n$, число ходов $z$ ). Кроме того, результатами расчета являются скорость движения в трубках КТ жидкости $w_{2}$ и пара $w_{1}$ (м/с), потери давления $\Delta P_{1}, \Delta P_{2}$ (Па) и коэффициенты гидравлического трения $\xi_{1}$ и $\xi_{2}$.

Исходными данными для расчета регулирующего органа (РО) являются $m_{1}, P s$, шероховатость труб $k_{e}$ (мм), длина паропровода Ls. Результатами расчета являются $K_{v}$ по ГОСТ, максимальный расход для выбранного РО $G_{\max }$, диапазон перемещения затвора РО $\Delta S$. 


\section{ТЕХНІЧНІ ЗАСОБИ ТА ІНФОРМАЦІЙНІ ТЕХНОЛОГІЇ В СИСТЕМАХ УПРАВЛІННЯ}

Результатами расчета параметров модели динамики являются коэффициенты $K_{1}, K_{2}, K_{3}, K_{4}, K_{5}, K_{6}, K_{7}$ и постоянные времени $T_{L}, T_{S}, T_{T}$.

\section{Конструктивный расчет и расчет статики}

Блок схема алгоритма представлена на рисунке 2. Расчет проводится на основании методики [8], которая нами усовершенствована. Произвольно заданные параметры теплообменника и скорость течения жидкости в результате многократного выполнения блока основного расчета, работа которого представлена на рисунке 3, итеративно приближаются к оптимальным и внутренне непротиворечивым.

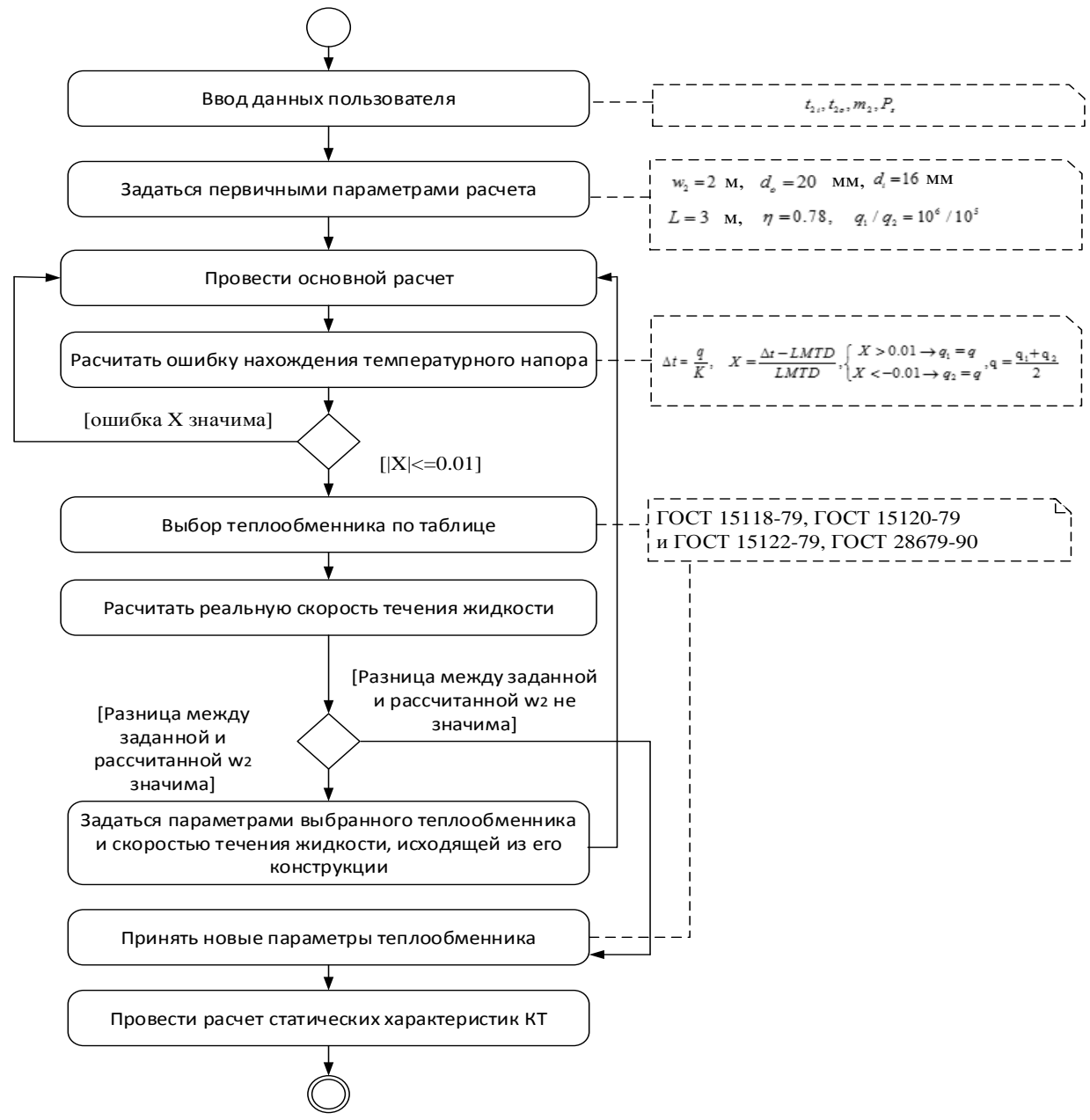

Рис. 2 - UML блок-схема алгоритма расчета KT

Расчет математической модели динамики

Для построения модели динамики используется модифицированная нами методика расчета, основанная на приведенной в [9]. 


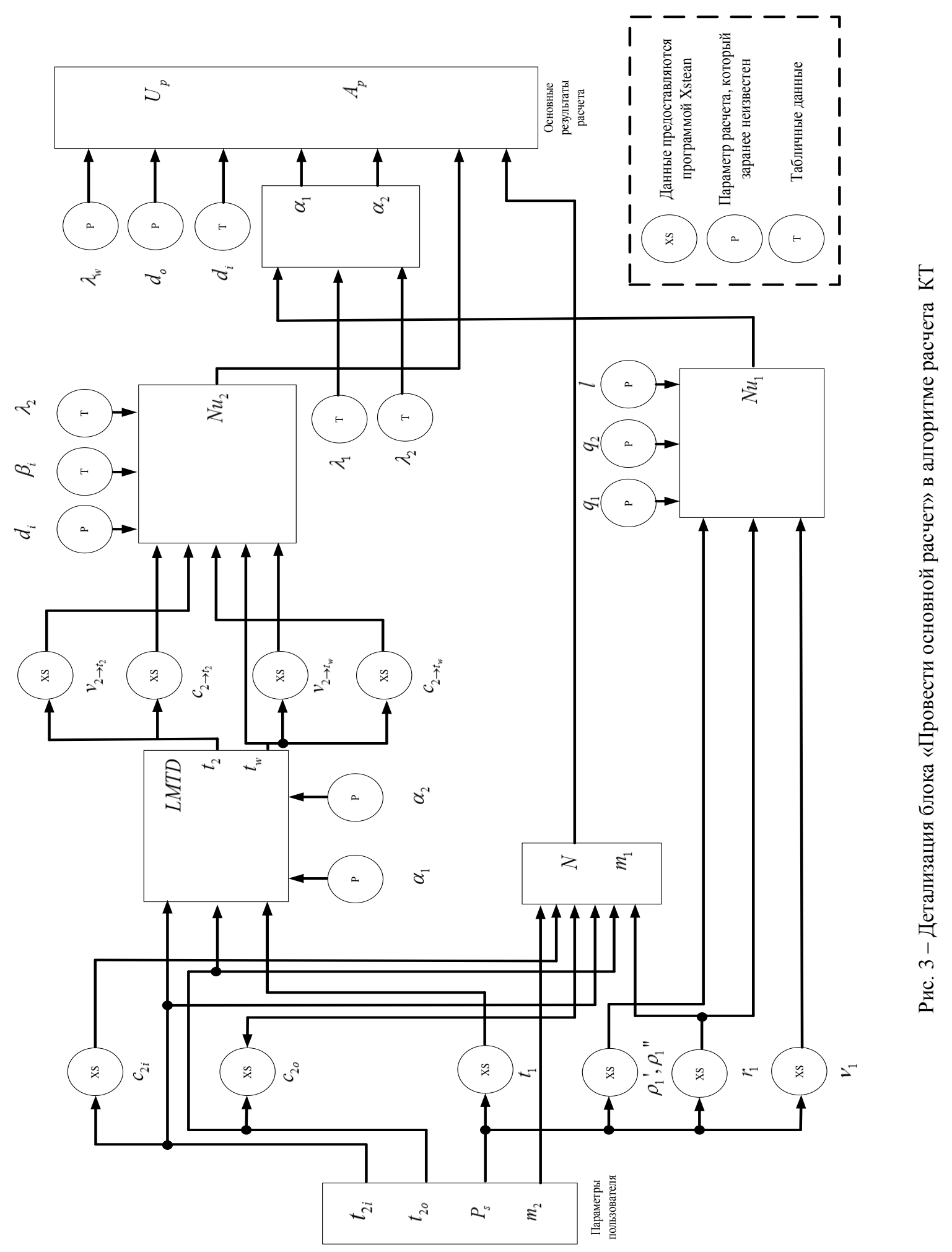




\begin{tabular}{|l|l|}
\hline $\begin{array}{l}k_{1} \approx 1 \\
k_{2}=\frac{1-k_{3}}{k_{3}}, k_{3}=\frac{\alpha_{2} \cdot S_{2}}{\alpha_{2} \cdot S_{2}+m_{2} \cdot C_{t 2}}\end{array}$ & $W_{2}=\frac{\rho_{2} \cdot L \cdot n \cdot z \cdot\left(d_{o}{ }^{2}-d_{o}{ }^{2}\right) \cdot \pi}{4}$ \\
$k_{4}=\frac{m_{2} \cdot C_{t 2}}{a_{2} \cdot S_{2}+m_{2} \cdot C_{t 2}}$ & $W_{T}=\frac{\rho_{\text {steel }} \cdot L \cdot n \cdot z \cdot\left(d_{o}{ }^{2}-d_{i}{ }^{2}\right) \cdot \pi}{4}$ \\
$k_{5}=\frac{C_{t 2 i} t_{2 i}-C_{t 2 o} t_{2 o}}{a_{2} \cdot S_{2}+m_{2} \cdot C_{t 2}}$ & $W_{1}=\frac{\rho_{1} \cdot L \cdot\left(D_{o}{ }^{2}-n \cdot d_{o}{ }^{2}\right) \cdot \pi}{4}$ \\
\cline { 2 - 2 }$k_{6}=\frac{\left(t_{2 i}+\frac{r_{1 \rightarrow t_{1}} \cdot m_{1}}{C_{t_{2}} \cdot m_{2} \cdot \eta}\right)-\left(t_{2 i}+\frac{r_{1 \rightarrow t_{1}} \cdot m_{1} \cdot 0.95}{C_{t_{2}} \cdot m_{2} \cdot \eta}\right)}{m_{1}-\left(m_{1} \cdot 0.95\right) \cdot k_{7} \cdot k_{1} \cdot k_{3} /\left(1-\mathrm{k}_{2} \cdot k_{3}\right)}$ & $S_{1} \approx S_{2}=n \cdot z \cdot L \cdot \pi \cdot d_{o}$ \\
$k_{7}=\frac{\partial t}{\partial P_{1}}$ & $T_{L}=\frac{W_{2} \cdot C_{t 2}}{a_{2} \cdot S_{2}+m_{2} \cdot C_{t 2}}, T_{S}=\frac{m_{1}}{W_{1}}$ \\
& $T_{T}=\frac{W_{T} \cdot c_{T}}{a_{1} \cdot S_{1}+a_{2} \cdot S_{2}}$ \\
\end{tabular}

Структурная схема линейной математической модели динамики приведена на рис. 4.

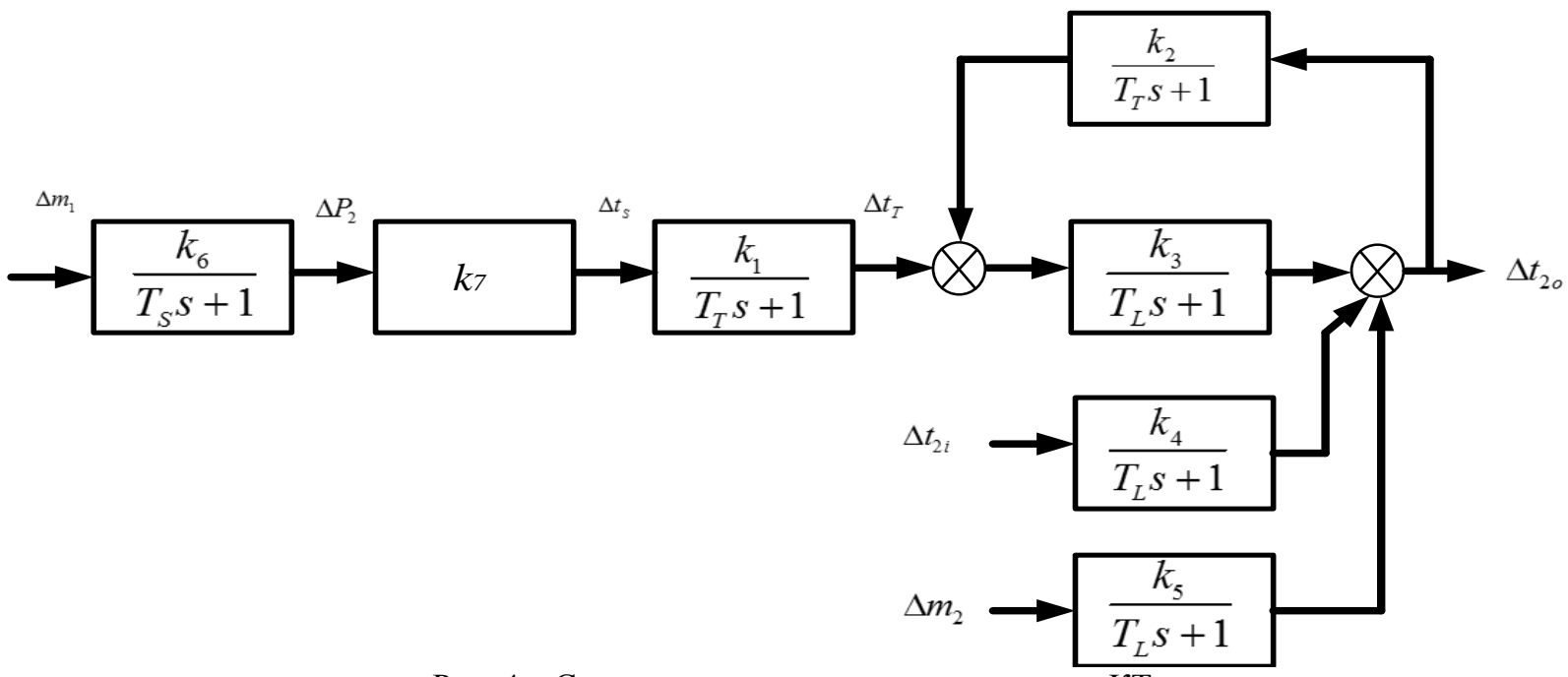

Рис. 4 - Структурная схема модели динамики КТ

Разработка системы автоматического управления

Схема связи подсистем в Simulink-модели САУ представлена на рис. 5.

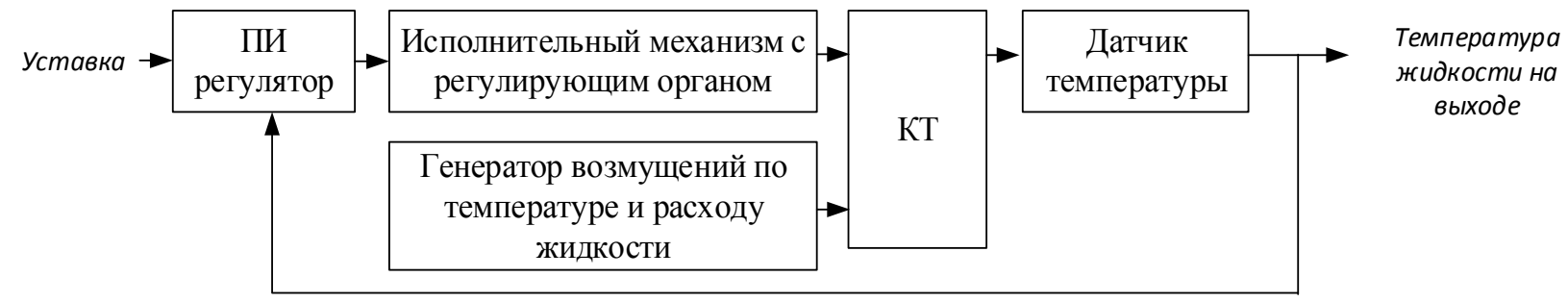

Рис. 5 - Схема связи подсистем в Simulink-модели САУ 


\section{ТЕХНІЧНІ ЗАСОБИ ТА ІНФОРМАЦЙНІ ТЕХНОЛОГІЇ В СИСТЕМАХ УПРАВЛІННЯ}

Задавая требуемые параметры в блоки подсистем, пользователь может проводить моделирование динамики замкнутой САУ, проверять её работу при заданных возмущения и/или при отклонении параметров модели. Подсистема датчика температуры складывается из передаточной функции, моделирующей инерционность датчика и его погрешность. Подсистема исполнительного механизма с регулирующим органом моделирует регулирующий орган с линейной характеристикой для САУ. Основной характеристикой исполнительного механизма для САУ является время перемещения $\mathrm{T}_{S M}$.

При вызове параметров блока КТ вызывается диалоговое окно, реализованное в Matlab GUIDE, которое позволяет пользователю ввести требуемые параметры и, в случае их корректности, получить в результате параметры модели статики и динамики. Пример диалогового окна с результатами расчета приведен на рисунке 6.

Для управления объектом используется стандартный блок Simulink PID Controller, который использует алгоритм автонастройки по частотным характеристикам. Был проведен эксперимент на проверку робастности рассчитываемого ПИ регулятора для КТ при изменении нагрузки на установку $(0.6,0.8,1.0,1.2$ от номинала).

Настройки определялись для регулятора при номинальной нагрузке и были проверены при изменении нагрузки (с соответствующим изменением модели). Переходные процессы, отображенные на рисунках 7 и 8 показывают, что полученная САУ является робастной как при управлении по возмущению, так и по заданию.

\section{Проверка результатов}

В качестве исходных возьмем данные, отображённые в параметрах диалогового окна на рис. 6. Сверка результатов конструктивных параметров расчета проведена с программой HTRI Xchanger Suite. B качестве параметров теплообменника программе были подставлены параметры теплообменника из таблицы ГОСТ.

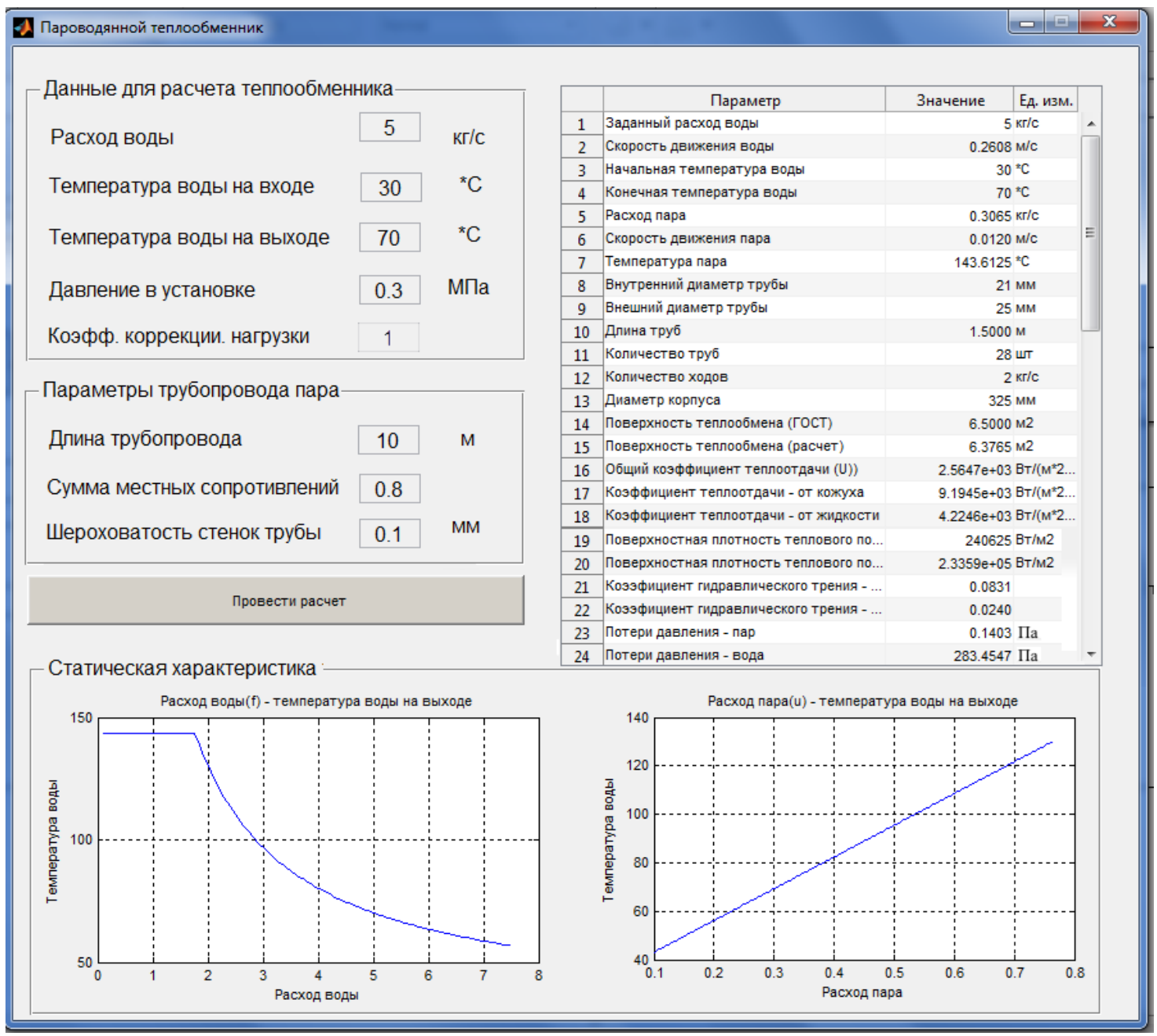

Рис. 6 - Диалоговое окно настройки параметров КТ в разработанном ПО 
Для сравнения полученных результатов используем расчет по программе Xchanger. Результаты расчета по разработанному ПО и программе приведены в табл. 2. Как видно, результаты расчета близки, хотя и не совпадают полностью. Это обусловлено различием алгоритмов расчета, различием конструкций теплообменников по ГОСТ и TEMА, а также различным подходом к вычислению среднего температурного напора, точное вычисление которого является сложной научной задачей, требующей множества экспериментальных данных.

Таблица 2 - Сравнение результатов конструктивного расчета КТ в разработанном ПО и Хchanger

\begin{tabular}{|c|c|c|c|c|}
\hline & $\mathrm{U}\left(\mathrm{BT} / \mathrm{M}^{2} * \mathrm{C}\right)$ & $\mathrm{N}(\mathrm{MBT})$ & $\mathrm{A}\left(\mathrm{M}^{2}\right)$ & Сp. темп. напор \\
\hline ПО & 2560 & $\begin{array}{l}\text { Tеор.:0.838 } \\
\text { Реал: } 0.654\end{array}$ & $\begin{array}{ll}\text { Расч: } & 6.38 \\
\text { Реал: } 6.5 & \\
\end{array}$ & LMTD - 92.17 \\
\hline $\begin{array}{l}\text { HTR } \\
\text { X } \\
\text { Xchanger }\end{array}$ & $\begin{array}{l}\text { Чистый: } 2147 \\
\text { Требуемый: } 1541\end{array}$ & 0.752 & $\begin{array}{c}\text { Реал: } \\
\text { Эфф: } 5.978\end{array}$ & EMTD - 81.91 \\
\hline
\end{tabular}

Сверка модели динамики была проведена с нелинейной моделью, описанной в [10]. Для сверки проведем подачу скачка, увеличив расход пара на 0.1 кг/с и снимем кривую разгона (рис. 7).

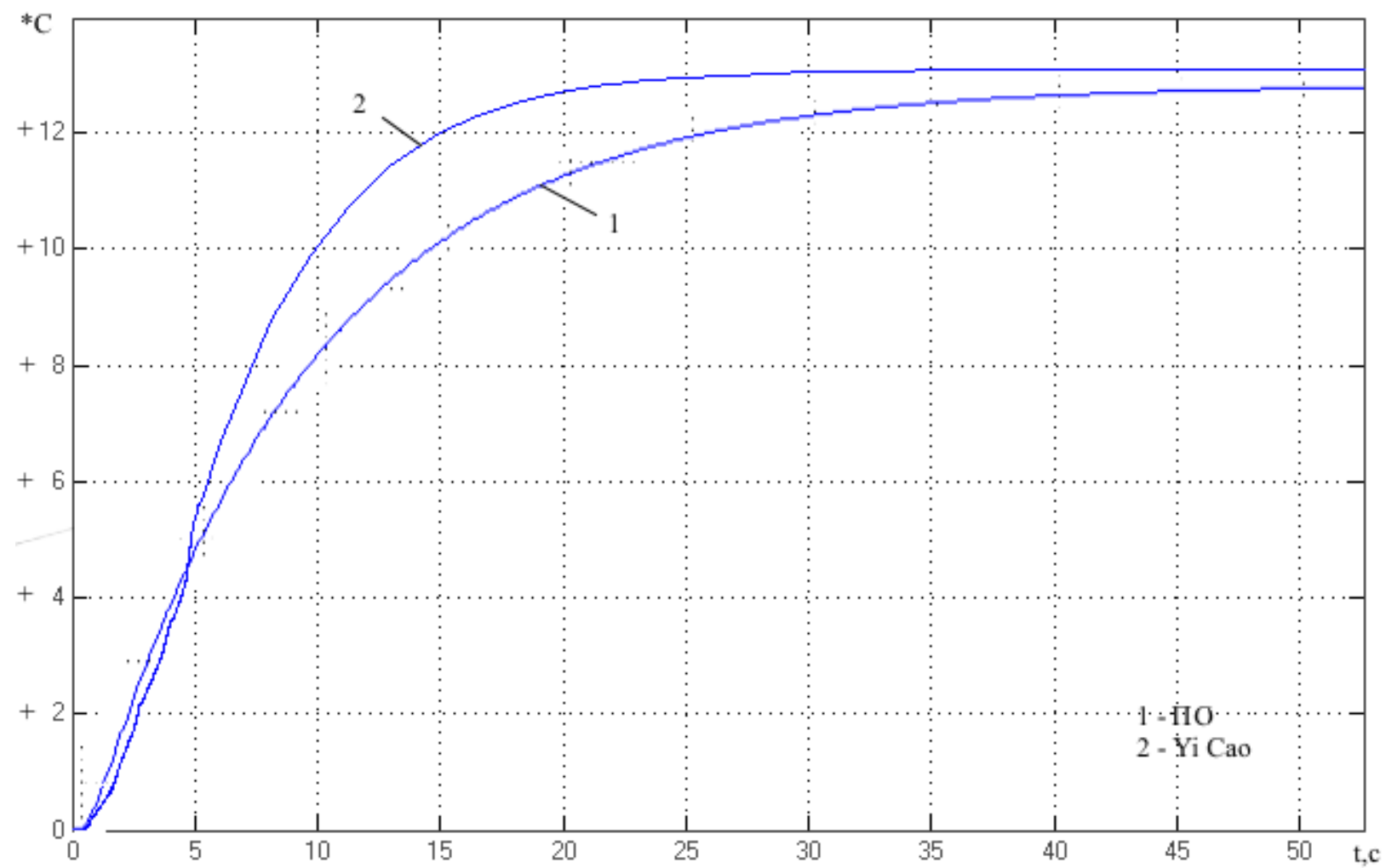

Рис. 7 - Сравнение кривых разгона моделей динамики КТ

Из рис.7 видно, что поведение моделей приблизительно одинаково, а причина различий состоит в учете нелинейных зависимостей в модели [10]. 
ТЕХНІЧНІ ЗАСОБИ ТА ІНФОРМАЦІЙНІ ТЕХНОЛОГІЇ В СИСТЕМАХ УПРАВЛІННЯ

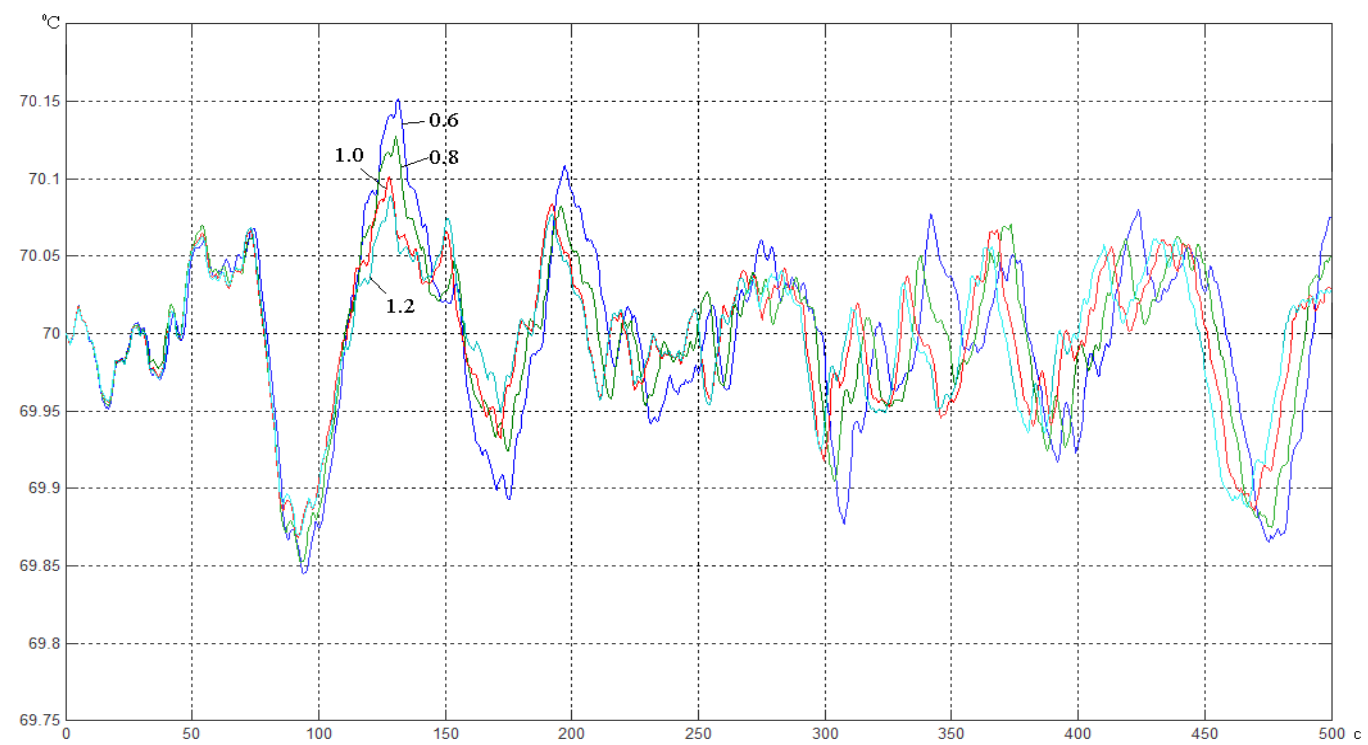

Рис. 8 - Переходные процессы при управлении по возмущению при разных нагрузках КТ

Сравнение переходных процессов при моделировании замкнутых систем автоматического управления с включением сравниваемых моделей и одинаковыми настройками регулятора (рис. 8, 9) показало высокую робастность разработанной системы.

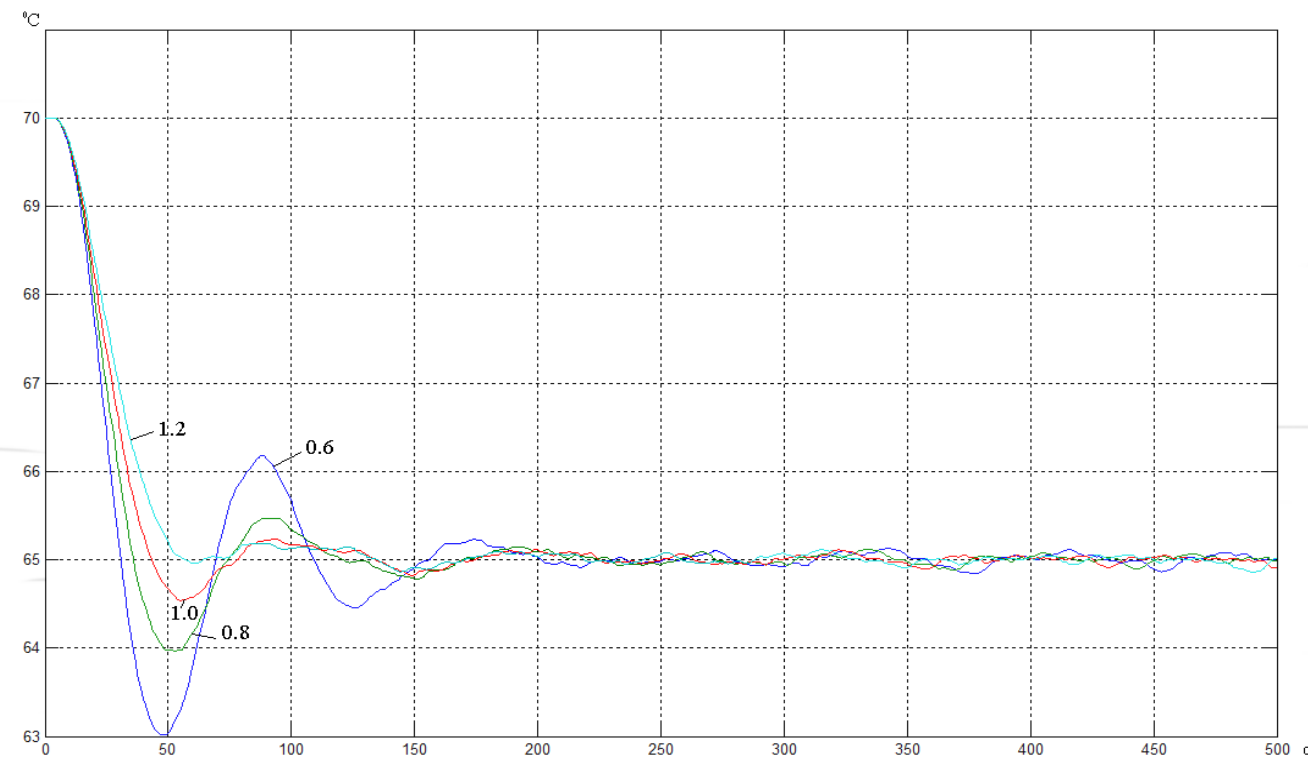

Рис. 9 - Переходные процессы при управлении по заданию при разных нагрузках КТ

\section{Выводы}

Алгоритм разработки современных робастных систем управления технологическими установками создаваемых технологических процессов должен включать несколько составляющих: расчет конструктивных параметров агрегата, определение диапазонов изменения параметров технологических потоков с выбором для них регулирующих органов и датчиков, аналитический расчет математической модели установки, расчет системы автоматического управления. Существующие на рынке программы технологического моделирования, которые представляют функциональные возможности такого расчета для ряда распространенных установок химикотехнологических процессов, оказались не способны выполнить расчет для таких широко распространенных аппаратов, как кожухотрубные парожидкостные теплообменники. Проведенный анализ показал необходимость разработки специализированного программного обеспечения для выполнения этой задачи. 
В качестве языка программирования при разработке программного обеспечения для расчета установок и систем управления выбран Mathworks Matlab, в том числе благодаря наличию большого количества пользователей и специализированных библиотек. В основу разработанного программного обеспечения положен алгоритм построения модели теплообменника, отличительной особенностью которого является минимальное число требуемых исходных данных, а именно начальная и конечная температуры жидкости, массовый расход жидкости и давление в паропроводе. Программное обеспечение позволяет выбрать стандартный теплообменник из ряда ГОСТ, провести расчет регулирующего органа на паропроводе, определить параметры статики и динамики теплообменника, провести синтез регулятора и проверить робастность замкнутой системы управления с расчётными настройками регулятора при изменении нагрузки теплообменника.

Результаты конструктивного расчета теплообменника сверены с результатами программы расчета параметров теплового расчета HTRI Xchanger Suite[5]. Результаты расчета являются близкими, отличия объясняются различием методики расчета и конструктивных особенностей теплообменников в стандарте ГОСТ и ТЕМА. Математическая модель динамики теплообменника сверена по кривым разгона с нелинейной математической моделью парожидкостного теплообменника, приведенной в [10]. При отличии принципов построения моделей, их кривые разгона в значительной степени совпадают.

Предполагается расширение функций разработанного программного обеспечения на расчет систем управления другими типами теплообменных аппаратов.

Результаты работы могут быть использованы для разработки высококачественных систем управления современных энергоэффективных технологических процессов в проектных и конструкторских организациях, учебном процессе, а также для изучения и совершенствования методов расчета параметров теплообменных аппаратов и математического моделирования их работы.

\section{Литература}

1. Гартман Т.Н. Основы компьютерного моделирования химико-технологических процессов/ Т.Н. Гартман, Д.В. Клушин.- М.: Академкнига, 2006;

2. Wilcox W.R. An example of sizing of a heat exchanger in which steam undergoes both a phase change and temperature change in the vapor and/or liquid// Chemical engineering design data, information and advice.- N.Y.: Clarkson University, 2007;

3. Wilcox W.R. Method for reboiler and condenser sizing using HYSYS or UniSim// Chemical engineering design data, information and advice.- N.Y.: Clarkson University, 2009;

4. Fritzon P. Object-oriented modeling and simulation with MODELICA 2.1.- N.Y.: John Wiley and Sons, 2004.

5. HTRI: Design Manual.- Texas: Heat transfer research Inc, 2006;

6. Справочник по теплообменникам /под ред. Э.Г. Мартыненко.- М.: Энергоатомиздат, 1987. - Т.2;

7. XSteam- Properties for water and steam.- Режим доступа: http://xsteam.sourceforge.net/.-Название с экрана;

8. Нікульшин В.Р. Тепломасообмін. /В.Р. Нікульшин, Л.П. Андрєєв, А.М. Бундюк .- Одеса: ОДПУ, 1999;

9. Демченко В.А. Автоматизация и моделирования технологических процессов АЭС и ТЭС.- Одесса: Астропринт, 2001;

10. Cao Yi. Dynamic modeling of steam condenser.- Cranfield (U.K.): Cranfield University, 2008.

\section{References}

1. Gartman T.N. Osnovy komp'yuternogo modelirovaniya ximiko-texnologicheskix processov/ T.N. Gartman, D.V. Klushin.- M.: Akademkniga, 2006;

2. Wilcox W.R. An example of sizing of a heat exchanger in which steam undergoes both a phase change and temperature change in the vapor and/or liquid// Chemical engineering design data, information and advice.- N.Y.: Clarkson University, 2007;

3. Wilcox W.R. Method for reboiler and condenser sizing using HYSYS or UniSim// Chemical engineering design data, information and advice.- N.Y.: Clarkson University, 2009;

4. Fritzon P. Object-oriented modeling and simulation with MODELICA 2.1.- N.Y.: John Wiley and Sons, 2004;

5. HTRI: Design Manual.- Texas: Heat transfer research Inc, 2006;

6. Spravochnik po teploobmennikam /pod red. E.G. Martynenko.- M.: Energoatomizdat, 1987. - T.2;

7. X Steam - Properties for water and steam.- Access mode: http://xsteam.sourceforge.net/.-Title from display;

8. Nikulshyn V.R. Teplomasoobmin/V.R. Nikul'shyn, L.P. Andrjejev, A.M. Bundjuk .- Odesa: ODPU, 1999;

9. Demchenko V.A. Avtomatizaciya i modelirovaniya texnologicheskix processov AES i TES.- Odessa: Astroprint, 2001;

10. Cao Yi. Dynamic modeling of steam condenser.- Cranfield (U.K.): Cranfield University, 2008. 